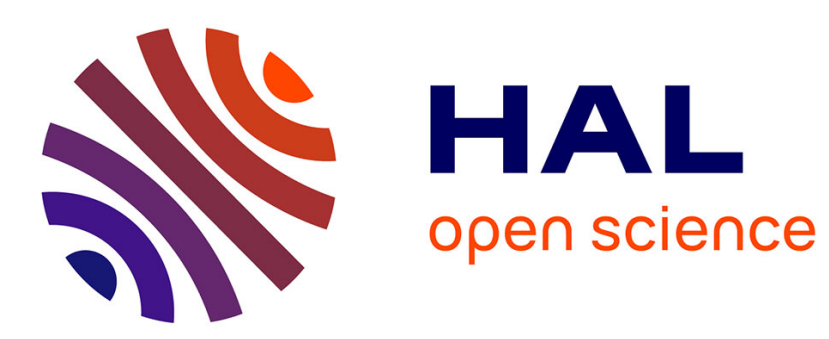

\title{
Chaque mois, des nouvelles de la recherche en logistique et SCM
}

Nathalie Fabbe-Costes

\section{To cite this version:}

Nathalie Fabbe-Costes. Chaque mois, des nouvelles de la recherche en logistique et SCM. Supply Chain Magazine, 2012, №61, Janvier-Février 2012, pp.101-102. hal-01424026

\section{HAL Id: hal-01424026 \\ https://hal-amu.archives-ouvertes.fr/hal-01424026}

Submitted on 1 Jan 2017

HAL is a multi-disciplinary open access archive for the deposit and dissemination of scientific research documents, whether they are published or not. The documents may come from teaching and research institutions in France or abroad, or from public or private research centers.
L'archive ouverte pluridisciplinaire HAL, est destinée au dépôt et à la diffusion de documents scientifiques de niveau recherche, publiés ou non, émanant des établissements d'enseignement et de recherche français ou étrangers, des laboratoires publics ou privés. 


\title{
Chaque mois, des nouvelles de la recherche en logistique et SCM
}

\author{
C'est avec un grand plaisir que j'inaugure la nouvelle rubrique de Supply Chain \\ Magazine intitulée « Le billet du Cret-Log ». Ce premier billet a pour but \\ de vous présenter l'objectif général de la rubrique, son ambition et les principes \\ rédactionnels que nous avons retenus en accord avec la rédaction de Supply Chain \\ Magazine. Notre principale mission est de contribuer au dialogue recherche - \\ entreprises, en vous donnant des nouvelles de la recherche en logistique et Supply \\ Chain Management (SCM). Ces « nouvelles " ne concerneront pas que les travaux \\ menés au Cret-Log, mais plus largement l'actualité de la recherche dans les \\ domaines qui intéressent les lecteurs de Supply Chain Magazine.
}

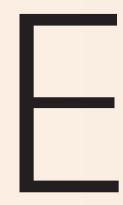
n tant que Directrice du Cret-Log depuis maintenant deux ans, je me réjouis d'engager mon équipe de recherche, avec l'aide d'Aurélien Rouquet, membre du Cret-Log et professeur à Reims Management School, qui assurera la coordination de la rubrique, dans une œuvre difficile et parfois ingrate de diffusion des connaissances académiques auprès d'un public de professionnels.

\section{Contribuer au dialogue \\ recherche - entreprises}

A l'heure où les centres de recherche et les entreprises sont appelés à collaborer dans le cadre de projets de recherche, où les Prides (Pôles Régionaux d'Innovation et de Développement Economique Solidaire) et les pôles de compétitivité œuvrent au rapprochement de ces organisations et où l'Etat, via ses ministères ou ses agences (exemple : ANR, ANRT, Ademe, Oseo), mais aussi l'Europe, soutiennent des programmes de recherche ambiticux (comme les projets autour du Grand Emprunt ou du 7c PCRD - Programme Cadre de Recherche et Développement : www.france-europe-innovation.fr/le-7eme-pcrd.php) destinés à soutenir l'effort d'innovation des entreprises, il nous semble important de contribuer à notre manière à ce rapprochement. Développer le

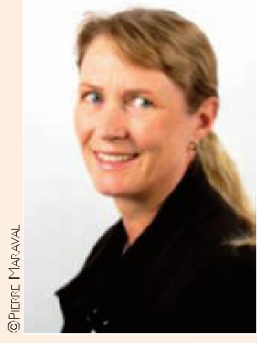

Nathalie Fabbe-Costes

Professeur des Universités

Directrice du Cret-Log (Centre de Recherche

sur le Transport et la LOGistique)

Aix-Marseille Université (AMU)

nathalie.fabbe-costes@univ-amu.fr dialogue recherche-entreprises va d'autant moins de soi que les dispositifs d'évaluations de la recherche n'incitent pas à ce dialogue, ni ne le valorisent. Les résultats produits par la recherche académique restent par ailleurs trop souvent " confidentiels ", du fait d'une diffusion sous forme de publications dont les styles et les formats sont peu accessibles aux lecteurs non avertis. La vulgarisation n'a malheureusement plus le vent en poupe ! C'est pourtant en informant les professionnels de nos programmes de recherche et des résultats que nous produisons que nous faciliterons la rencontre des projets des deux communautés et la coproduction de savoirs scientifiquement valides et pertinents pour l'action.

\section{Donner un écho du travail des chercheurs}

Le Cret-Log est une équipe de recherche spécialisée en logistique et qui défend depuis sa création en 1973 une ligne de recherche en lien avec les entreprises et les institutions. Fidèle à cette logique, le laboratoire a décidé de s'engager avec Supply Chain Magazine à animer une rubrique qui vise à donner aux lecteurs de la revue des "nouvelles de la recherche " en logistique et SCM. Telle est l'ambition du "Billet du Cret-Log ": donner dans chaque numéro de Supply Chain Magazine un écho du travail des chercheurs, en facilitant l'articulation entre deux catégories de professionnels - les chercheurs et les Managers - qui ont un intérêt mutuel à développer des collaborations. Volontairement courts et dans un style accessible pour des non-spécialistes, nos billets chercheront à faire partager les recherches et à faciliter les contacts entre chercheurs et Managers. Chaque billet sera en effet signé par son (ou ses) auteur(s) avec le contact courriel pour favoriser les échanges. L'ensemble de la production sera coordonné par Aurélien 
Rouquet, qui peut être contacté à aurelien.rouquet@reims-ms.fr.

\section{Cinq types de contenu}

Compte tenu de l'importance de la formation doctorale au Cret-Log, certains billets, rédigés par de jeunes docteurs en sciences de gestion, présenteront une synthèse des résultats de leur recherche doctorale. Ce sera le cas de prochains billets rédigés par Frédéric Pellegrin-Romeggio et par Elodie Kacioui-Maurin qui ont respectivement soutenu leur thèse en septembre et novembre 2011.

D'autres billets présenteront les résultats de programmes de recherche en cours ou achevés. Ce sera le cas pour plusieurs recherches actuellement menées dans le cadre du Programme de recherche et d'innovation dans les transports terrestres Predit 4 (www.predit.prd.fr/predit $4 /$ ) sur des thèmes comme la mutualisation, la veille logistique durable ou les achats durables.

Dans la mesure où les membres du Cret-Log participent à de nombreux colloques internationaux de recherche en logistique, management des opérations ou SCM que ce soit dans les réseaux francophones (RIRL), européens (Euroma), nordiques (Nofoma) ou britanniques (LRN), nous proposerons aussi des synthèses des recherches présentées en mettant en évidence les thèmes qui mobilisent les chercheurs, ainsi que les « nouveautés ". L'objectif sera ici de contribuer, audelà des travaux menés par l'équipe du Cret-Log, à un effort de veille.

Nous participerons aussi à un nécessaire effort de clarification de certaines notions de logistique et SCM problématiques ou polémiques dans une perspective de compréhension des enjeux pour les entreprises.

Enfin certains billets pourront revenir avec un prisme universitaire sur l'analyse d'un point d'actualité particulièrement important et qui mérite d'être analysé avec une certaine prise de recul.

\section{Une dimension appliquée} qui intéresse les Managers

Au-delà de sa vocation purement académique, la recherche en Supply Chain Management a en effet une dimension appliquée qui intéresse les Managers. Elle permet d'anticiper les transformations à l'œuvre dans les Supply Chains, aborde de nouvelles questions et développe de nouveaux concepts logistiques qui préfigurent de futures évolutions, propose des évaluations rigoureuses des stratégies et pratiques existantes, démystifie certaines idées reçues et invite à voir autrement. Autant de bonnes raisons pour nous lire chaque mois et pour échanger avec les auteurs des futurs billets!

\section{Le Cret-Log}

Avec plus d'une quarantaine d'enseignants-chercheurs et chercheurs rattachés au laboratoire et plus d'une trentaine de doctorants en formation à et par la recherche, le CretLog, qui a été le premier centre de recherche français à se spécialiser au début des années 1980 en logistique, fait aujourd'hui partie des centres de recherche européens réputés dans ce domaine.

Le Cret-Log développe des recherches autour de trois thématiques principales : le management logistique et le Supply Chain Management, le management des canaux de distribution et les stratégies inter-organisationnelles. II développe par ailleurs dans ces trois domaines des travaux en lien avec le développement durable. (schéma ci-contre)

Avec une production de sept thèses en moyenne par an, c'est un des centres les plus actifs en France pour la formation d'enseignants-chercheurs mais aussi de professionnels de haut niveau formés par la recherche. Notre formation doctorale attire des étudiants du monde entier. Pas moins de 18 nationalités sont représentées dans l'effectif des anciens docteurs comme des doctorants actuels.

Les chercheurs du Cret-Log publient dans les revues académiques, participent aux colloques de référence et contribuent à des ouvrages individuels ou collectifs. Ils participent aussi régulièrement à des manifestations professionnelles.

Enfin l'Université d'Aix-Marseille propose l'offre de formation en logistique la plus complète de France, formations qui sont toutes adossées au Cret-Log et bénéficient de l'expertise de ses membres : du DUT à la Licence Professionnelle à l'IUT d'Aix-en-Provence, et du Master au Doctorat à la Faculté d'Economie et de Gestion.

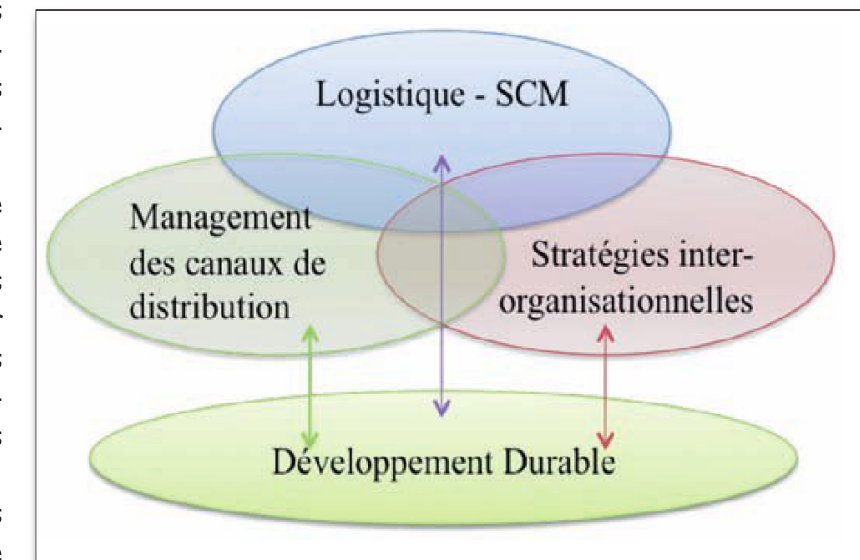

